\title{
Rapid and Visual Identification of Chlorophyllum molybdites With Loop-Mediated Isothermal Amplification Method
}

\author{
Nan Wang', Zhiyong Zhao', Jie Gao', Enjing Tian', Wenjie Yu' ${ }^{1}$, Hui Li', Juan Zhang', \\ Ruibin Xie', Xiaoyan Zhao' and Ailiang Chen ${ }^{1 *}$ \\ ${ }^{1}$ Institute of Quality Standard and Testing Technology for Agro-Products, Key Laboratory of Agro-product Quality and Safety, \\ Chinese Academy of Agricultural Sciences, Beijing, China, ${ }^{2}$ Institute for Agri-food Standards and Testing Technology, \\ Shanghai Academy of Agricultural Sciences, Shanghai, China, ${ }^{3}$ Institute of Mycology, Engineering Research Center \\ of Chinese Ministry of Education for Edible and Medicinal Fungi, Jilin Agricultural University, Jilin, China
}

OPEN ACCESS

Edited by: Lin Lin,

Jiangsu University, China

Reviewed by: Julien Reboud,

University of Glasgow,

United Kingdom

Ehsan Aryan,

Mashhad University of Medical

Sciences, Iran

Jianping Xu,

McMaster University, Canada

*Correspondence: Ailiang Chen ailiang.chen@gmail.com

Specialty section: This article was submitted to

Food Microbiology, a section of the journal

Frontiers in Microbiology

Received: 06 December 2020 Accepted: 10 February 2021

Published: 18 March 2021

Citation:

Wang N, Zhao Z, Gao J, Tian E, Yu W, Li H, Zhang J, Xie R, Zhao X and Chen A (2021) Rapid and Visual Identification of Chlorophyllum molybdites With Loop-Mediated Isothermal Amplification Method.

Front. Microbiol. 12:638315. doi: 10.3389/fmicb.2021.638315
Chlorophyllum molybdites is a kind of common poisonous mushroom in China that is widely distributed in different areas. Food poisoning caused by accidentally eating C. molybdites has become more frequent in recent years. In 2019, there were 55 food poisoning incidents caused by eating this mushroom in China. Mushroom poisoning continues to be a common health issue of global concern. When mushroom poisoning occurs, an effective, simple, and rapid detection method is required for accurate clinical treatment or forensic analysis. For the first time, we established a loop-mediated isothermal amplification (LAMP) assay for the visual detection of $C$. molybdites. A set of specific LAMP primers was designed, and the specificity was confirmed against 43 different mushroom species. The LAMP method could detect as low as 1 pg of genomic DNA. Boiled mushrooms and artificial gastric-digested mushroom samples were prepared to test the applicability of the method, and the results showed that as low as 1\% C. molybdites in boiled and digested samples could be successfully detected. The LAMP method can also be completed within $45 \mathrm{~min}$, and the reaction results could be directly observed based on a color change under daylight by the naked eye. Therefore, the LAMP assay established in this study provides an accurate, sensitive, rapid, and low-cost method for the detection of $C$. molybdites.

Keywords: poisonous mushroom, loop-mediated isothermal amplification, Chlorophyllum molybdites, ITS, on-site rapid detection

\section{INTRODUCTION}

As a larger fungus, mushrooms are widely distributed worldwide with high nutritional value and pharmacological effects (Lei et al., 2020). According to statistics, there are currently more than 14,000 species of mushrooms in the world, and more than 3,800 species are known in China, of which approximately 480 are poisonous (Wu et al., 2019). Many edible mushrooms have similar morphological characteristics to poisonous mushrooms, and thus, it is difficult to distinguish poisonous mushrooms by the naked eye (Yang, 2013). In China, many food poisoning incidents 
are caused by poisonous mushrooms every year (Chen et al., 2014; Sun et al., 2018). According to statistics, there were 276 independent mushroom poisoning incidents in 2019, involving 769 patients from 17 provinces and 22 deaths, with an overall mortality rate of $2.86 \%$ (Haijiao et al., 2020). Among them, Chlorophyllum molybdites is a typical type of mushroom that is prone to be eaten by mistake. C. molybdites is widely distributed throughout the year in different regions of China, which has become the most poisonous mushrooms closest to humans (Soltaninejad, 2018). In 2018, 55 mushroom poisoning incidents caused by accidental eating of C. molybdites involving at last 133 patients were reported in China (Soltaninejad, 2018).

The mushroom poisoning incidents have become one of the serious food safety issues in China. For different types of poisonous mushrooms, they contain various toxins that result in different kinds of toxic symptoms. Mis-eating of C. molybdites mainly causes serious gastrointestinal discomfort that can last from 1 to $6 \mathrm{~h}$ after consumption. The toxin is a protein called molybdophyllysin (Yamada et al., 2012). If not treated in time, it will cause multiple organ failure and is life-threatening. Therefore, the rapid and accurate identification of mushroom species is necessary for proper clinical treatment. In food poisoning incidents caused by poisonous mushrooms, it has been found that mushroom samples lose their original morphological features after being cooked and digested. Therefore, identifying mushrooms by morphological identification methods is impossible. Various methods for mushroom species identification have been developed, in which the detection of mushroom toxins and DNA-based molecular biology methods are the most widely used (Li et al., 2016). With the discovery of various toxins in mushrooms, methods for identifying mushroom species by detecting toxins have been developed, such as chromatography, liquid chromatography diode array detection, inductively coupled plasma atomic emission spectrometry, and other technologies (Ahmed et al., 2010; Yao et al., 2018; Wurita et al., 2019; Numanoglu Cevik, 2020). However, most of these methods require expensive instruments, a long detection cycle, and high detection costs, which are not suitable for application in primary regions. The rapid detection of toxins represented by lateral flow immunoassays has the advantages of low cost, rapid detection, and intuitive results, but the toxin antibodies are not stable and are difficult to prepare, and the sensitivity and specificity need to be improved for practical applications, especially with respect to processed products (Tripathi et al., 2018). As the basis of the genetic material of organisms, nucleic acid would not be affected by age, season, developmental stage, and environment factors, and has a high thermal stability that could not be interfered by alterations during processing (Hebert et al., 2003; Garnica et al., 2016). With the development of polymerase chain reaction (PCR), a variety of diagnostic methods have been derived, such as real-time PCR, polymerase chain reaction-restriction fragment length polymorphism (PCR-RFLP), and DNA barcoding (Sugano et al., 2017; Kondo et al., 2019; Wu et al., 2019). However, these methods usually rely on expensive instruments and are timeconsuming, which were technically challenging to achieve on-site rapid detection. Therefore, the development of simple, efficient, accurate, and sensitive mushroom species identification methods would be helpful for timely symptomatic treatment in the early onset of poisoning.

Isothermal amplification methods have been widely used for species identification of pathogenic fungi in recent years (He et al., 2019; Qiu et al., 2019; Bodulev and Sakharov, 2020). Among them, loop-mediated isothermal amplification (LAMP) is the most widely used one, which has received increasing attention (Lee, 2017). LAMP was first proposed by Notomi et al. (2000). The LAMP reaction contains a set of four to six specific primers, which can quickly identify six to eight regions on the target sequences and bind to strand displacement active DNA polymerase. LAMP reaction can be carried out at a constant temperature $\left(60-65^{\circ} \mathrm{C}\right)$, generating a vast number of amplicons (Lee, 2017). There are various detection methods for LAMP products; among them, the colorimetric method is easily used in on-site detection. The reaction result of the colorimetric method is visible to the naked eye, and it can be realized by only relying on a water bath without expensive equipment (Wang et al., 2014). After more than 20 years of development, LAMP has been widely used in the field of pathogenic microorganism rapid text (Lv et al., 2020), clinical disease diagnosis (Wang W.T. et al., 2019), food hygiene inspection (Zhu and Wang, 2018), and environmental monitoring (Becherer et al., 2020), in view of its efficient, specific, and sensitive detection capabilities, as well as a wide range of applications and the capacity of this technology to be performed outside of the routine laboratory environment. In terms of mushroom species identification, Vaagt et al. (2013) first applied the LAMP-based technique to the detection of Amanita phalloides. He et al. (2019) established the LAMP method for 10 poisonous Amanita detections. To date, there is no report on the detection and identification of C. molybdites.

In this study, the LAMP assay based on the colorimetric method targeting the internal transcribed spacer (ITS) was established for the rapid detection of C. molybdites from edible mushrooms with similar shapes and other common toxic mushrooms. In addition, considering the practical application of this method, mushroom processing and digestion in the human body were simulated. The LAMP detection method of C. molybdites established in this study is of great significance for the rapid identification of the toxic mushrooms in poisoning events, the targeted treatment after poisoning, and the prevention and diagnosis of poisoning.

\section{MATERIALS AND METHODS}

\section{Sample Collection}

A total of 44 mushroom species were collected in this study, including 15 poisonous mushrooms (Figure 1). These mushrooms mainly include edible fungi similar to C. molybdites (e.g., Macrolepiota), common poisonous and edible poisonous fungi in China. Among them, the C. molybdites sample was collected from a vineyard in Shanghai, China, in June 2020; and the remaining 43 mushrooms were collected from Lijiang, Yunnan, China, in July to September 2020. All samples were collected with the entire sporocarps and were initially 


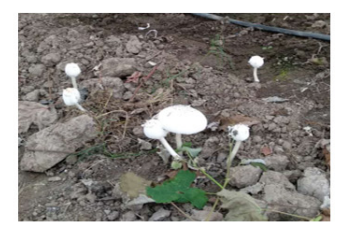

Chlorophyllum molybdites

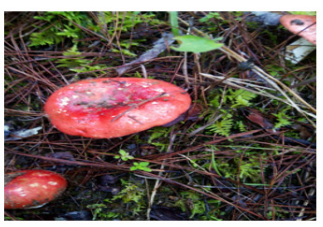

Russula rosacea

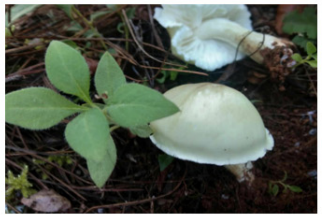

Chalciporus rubinellus

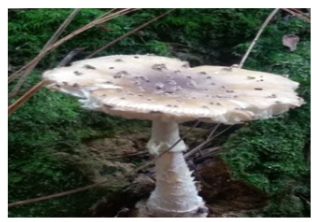

Amanita parvipantherina

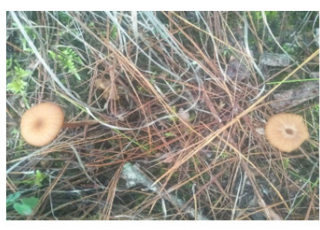

Laccaria aurantia

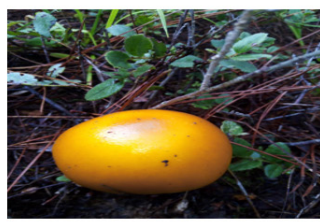

Amanita hemibapha

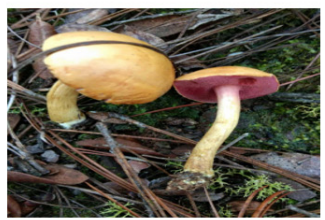

Chalciporus rubinellus

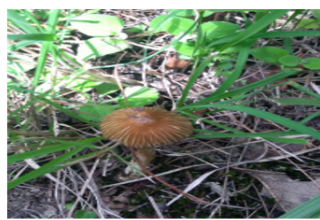

inocybe rimosa

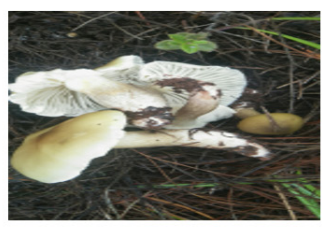

Russula variata

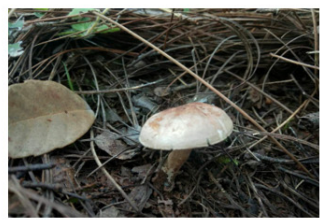

Hebeloma crustuliniforme

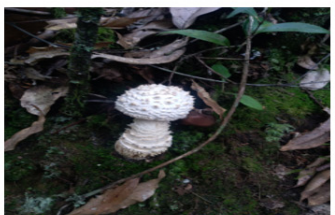

Amanita concentrica

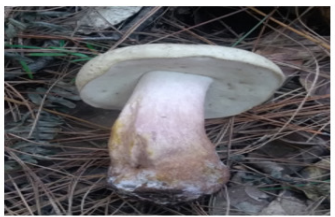

Tylopilus neofelleus

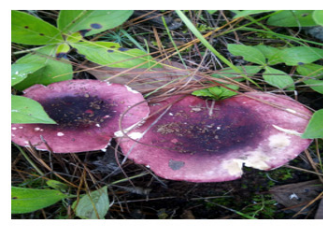

Russula velenovskyi

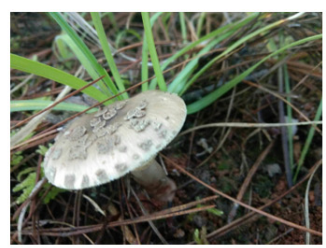

Amanita citrinoannulata

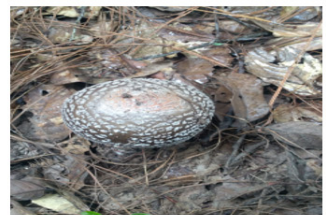

Amanita sepiacea

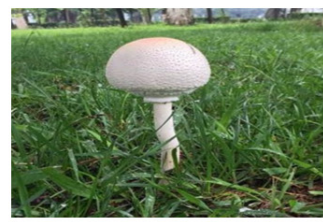

Macrolepiota dolichaula

FIGURE 1 | Some photographs of mushroom species collected in this study. Among them, Chlorophyllum molybdites was collected in Yunnan, China (2452' $\mathrm{N}$ and $\left.102^{\circ} 49^{\prime} \mathrm{E}\right)$, and the other mushrooms were collected from Shanghai, China ( $31^{\circ} 11^{\prime} \mathrm{N}$ and $\left.121^{\circ} 29^{\prime} \mathrm{E}\right)$. The picture of $C$. molybdites was taken by ZZ, and the remaining 15 photos of mushrooms were taken by $L$.

identified using morphological characteristics. Furthermore, DNA barcoding methods based on ITS genes were carried out to cross-confirm the identities of the above 44 mushrooms. All samples were stored at $-20^{\circ} \mathrm{C}$ for further analysis.

\section{DNA Extraction, PCR, and Sanger Sequencing}

All samples were rapidly dried by liquid nitrogen and then fully ground into powder. According to the manufacturer's instructions, the extraction of total genomic DNA was performed by a DNA secure plant kit (TIANGEN, China). The concentration and quality of DNA were measured by a Nanodrop 2000 ultramicro-spectrophotometer (Thermo Fisher Scientific, United States) and then diluted to $10 \mathrm{ng} / \mu \mathrm{L}$ for further study. A universal primer of ITS4 and ITS5 (White et al., 1989) was used for PCR amplification of the 44 mushrooms in a PCR thermal cycler (Applied Biosystems, United States). The PCR mixtures contained $12.5 \mu \mathrm{L}$ of $2 \times$ Taq PCR Master Mix, $0.3 \mu \mathrm{L}$ of each primer, $3 \mu \mathrm{L}$ of DNA, and $6.8 \mu \mathrm{L}$ of $\mathrm{ddH}_{2} \mathrm{O}$ in a total volume of $25 \mu \mathrm{L}$. Primer amplification of the ITS fragments was conducted in the following conditions: initial denaturation at $94^{\circ} \mathrm{C}$ for $5 \mathrm{~min}$, followed by 25 cycles of $94^{\circ} \mathrm{C}$ for $30 \mathrm{~s}, 58^{\circ} \mathrm{C}$ for $30 \mathrm{~s}$, and $72^{\circ} \mathrm{C}$ for $30 \mathrm{~s}$, and a final extension step at $72^{\circ} \mathrm{C}$ for $10 \mathrm{~min}$. Amplified PCR products were evaluated by electrophoresis on a $1.5 \%$ agarose gel and then sent to Beijing Sangon Biological Technology (China) for Sanger sequencing. The SeqMan program in the DNASTAR software was used to check and splice the two-way sequencing results to remove the low-quality region. The spliced sequences were used as queries for BLAST analysis in National Center for Biotechnology Information $\left(\mathrm{NCBI}^{1}\right)$ based on sequence similarity and comprehensive score. Finally, we submitted the obtained sequence to the GenBank database (accession numbers MW192451-MW192494).

\section{LAMP Primers Design}

Internal transcribed spacer gene sequences were chosen as the amplification target to design LAMP-specific primers of

\footnotetext{
${ }^{1}$ https://www.ncbi.nlm.nih.gov/
} 
C. molybdites. Eleven available ITS sequences of C. molybdites were downloaded from the NCBI GenBank database (accession numbers: KM609396.1, MK541937.1, MK541933.1, MK541934.1, MK541935.1, MK541936.1, MK541940.1, MK560059.1, MK541938.1, MK541941.1, MK541939.1). Then multiple sequence alignments were performed to seek the intraspecific conserved interval of $C$. molybdites, which served as the primer design region. To ensure the specificity of primers, the ITS sequences of the other 17 species of Chlorophyllum (C. neomastoideum, C. arizonicum, C. sphaerosporum, C. agaricoides, C. alborubescens, C. brunneum, C. demangei, C. globosum, C. hortense, C. levantinum, C. lusitanicum, C. nothorachodes, C. olivieri, C. palaeotropicum, C. pseudoglobosum, C. rhacodes, and C subrhacodes) and 43 mushrooms collected in this study were downloaded to align with C. molybdites using the MEGA software. Specific LAMP primers were designed in the interspecific differentiated and intraspecific conservative intervals.

A set of C. molybdites specific LAMP primer was designed by using GLAPD ${ }^{2}$ (Jia et al., 2019). For the multiple primer sets output by GLAPD, a theoretical verification for specificity of the LAMP primers was carried out by Primer-BLAST ${ }^{3}$ in NCBI. Then, we determined whether there are primer dimers, hairpins, false priming, and cross dimer using the Primer Premier version 5 software. Finally, validation experiments were conducted on each specific and good quality primer set to confirm the amplification efficiency and specificity. The primers with the highest amplification efficiency, good method stability, and specificity were selected as the LAMP-specific primers for this study. The selected LAMP primers contained two outer primers (C. mol-F3 and C. mol-B3), two inner primers (C. molFIP primer consisted of the complementary sequences of F1c and F2 and C. mol-BIP primer consisted of B1C and B2), and a loop primer (C. mol-LB). The position and sequences of the C. molybdites specific LAMP primer are shown in Figure 2 and Table 1.

\section{LAMP Reaction and Specificity Analysis}

The two pairs of outer primers, two inner primers, and loop primers were pre-diluted with $\mathrm{ddH}_{2} \mathrm{O}$ to 200,50 , and $200 \mu \mathrm{M}$ in concentration, respectively, and then they were mixed in a ratio of 3:10:96 $(\mathrm{V} / \mathrm{V} / \mathrm{V})$. LAMP reaction was conducted via a PCR thermal cycler (Applied Biosystems, United States) in a 10$\mu \mathrm{L}$ volume, which contained $5 \mu \mathrm{L}$ of WarmStart Colorimetric LAMP 2X Master Mix (New England Biolabs, United States), $1.09 \mu \mathrm{L}$ of primer mixtures, $2 \mu \mathrm{L}$ of the DNA template, and replenishing $\mathrm{ddH}_{2} \mathrm{O}$ to $10 \mu \mathrm{L}$. Amplification of the primers for the ITS fragment was carried out under the temperature of $65^{\circ} \mathrm{C}$ for $45 \mathrm{~min}$. After the reaction phase, we placed the reaction tubes on a white paper and then judged the reaction results according to the color change. The results of the positive LAMP were determined by direct visual observation of the change in color from pink to yellow in visible light.

\footnotetext{
${ }^{2}$ http://cgm.sjtu.edu.cn/GLAPD/online/

${ }^{3}$ https://www.ncbi.nlm.nih.gov/tools/primer-blast/
}

To assess the specificity of newly designed LAMP primers, genomic DNA from 43 mushrooms collected in this study were tested by the LAMP assay. If only the color of positive control (C. molybdites) turned to yellow, while the negative and blank control remained pink, the specificity of the primer set was good.

\section{LAMP Method Suitability Analysis}

To further verify the applicability of the LAMP method established in this study, we simulated the course of mushroom processing and body digestion process. A series of mushroom samples, including boiled and digested samples, were subjected to analysis by LAMP.

Mushrooms of C. molybdites and Pleurotus eryngii were mixed, respectively, in the final mass ratios of $1: 1,1: 10$, and 1:100 ( $\sim 100 \mathrm{mg}$ total mass) using the obtained powders. Prepared mushrooms of single $C$. molybdites and mixtures were boiled in water for $10 \mathrm{~min}$ at $100^{\circ} \mathrm{C}$, respectively. DNA extraction and LAMP reaction were performed as described above.

Artificial gastric fluid was made up of $0.05 \mathrm{~g}$ potassium chloride, $0.42 \mathrm{~g}$ sodium chloride, and $0.32 \mathrm{~g}$ pepsin in $100 \mathrm{~mL}$ water, and then the $\mathrm{pH}$ was adjusted to 2.0 with $2 \mathrm{~mol} / \mathrm{L}$ hydrochloric acid (Minekus et al., 2014). Boiled mushrooms of single C. molybdites and mixtures (C. molybdites and P. eryngii with the final mass ratios of $1: 1,1: 10$, and 1:100, respectively) were then incubated in artificial gastric fluid for $4 \mathrm{~h}$ at $37^{\circ} \mathrm{C}$, respectively. DNA extraction and LAMP reaction were performed as described above. In addition, the existence of human genes in the digestive tract, as well as other potential pollutants, such as the enterobacteria group, was considered. In order to further ensure the accuracy of the LAMP detection method established in this study, we conducted a specific verification experiment of LAMP primers on human saliva.

\section{RESULTS}

\section{Species Identification of Sanger Sequencing}

A total of 44 samples of genomic DNA were obtained. The DNA concentration of all samples was higher than $10 \mathrm{ng} / \mu \mathrm{L}$, and the ratio of 260/280 was between 1.8 and 2.2, which was enough to meet the requirements of the subsequent study. With the ITS gene as the amplification target, the true identities of 44 collected mushroom samples were further verified by Sanger sequencing technology. The PCR amplification and sequencing success rate of all samples was $100 \%$. The obtained sequences were blasted with the GenBank database from NCBI. The comparison results showed that ITS sequence similarity of all 44 species was $\geq 98 \%$ against the reference sequences, and the identification results confirmed the initial taxonomic assessment (Table 2). Subsequently, the ITS gene sequencing sequences of these 44 mushroom samples were uploaded to NCBI.

\section{Specificity and Sensitivity Analysis}

A reliable species-specific LAMP amplification primer set should display a high degree of specificity among various species and 
A
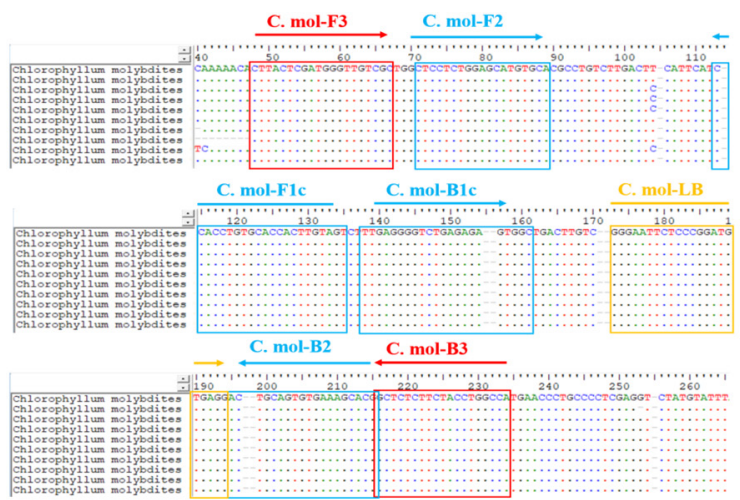

B

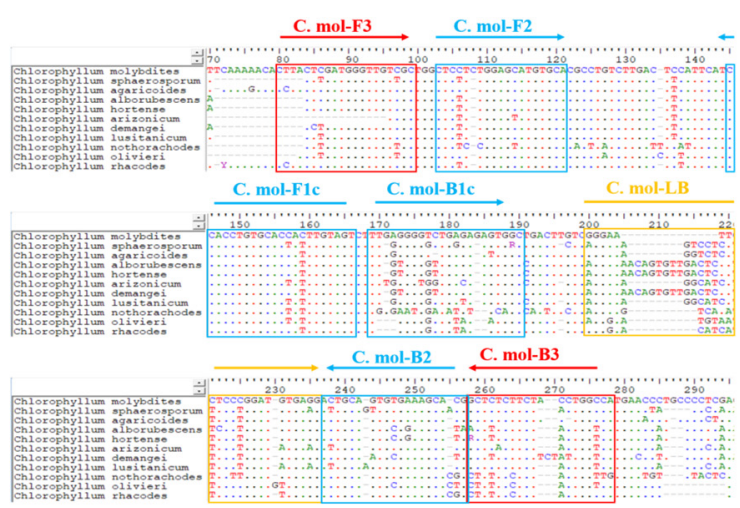

FIGURE 2 | Positions of the LAMP primer set of Chlorophyllum molybdites designed in the region of the ITS gene. (A) Multiple sequences alignment of homologous species of $C$. molybdites. (B) Multiple sequences alignment of related species of $C$. molybdites (not all shown in the figure).

TABLE 1 | The LAMP primer set of Chlorophyllum molybdites used in this study.

\begin{tabular}{ll}
\hline Primers & Sequence $\left(\mathbf{5}^{\prime} \mathbf{-} \mathbf{3}^{\prime} \mathbf{)}\right.$ \\
\hline C. mol-F3 & CTTACTCGATGGGTTGTCGC \\
C. mol-FIP & ACTACAAGTGGTGCACAGGTGGCTCCTCTGGAGCATGTGCA \\
C. mol-BIP & TTGAGGGGTCTGAGAGAGTGGCCGTGCTTCACACTGCAGT \\
C. mol-B3 & TGGCCAGGTAGAAGAGAGC \\
C. mol-LB & GGGAATTCTCCCGGATGTGAGG
\end{tabular}

should be highly conserved within the same species. Genomic DNA from 43 mushrooms was used to evaluate the specificity of the C. molybdites LAMP primer set designed in this study. For the LAMP assay, as shown in Figure 3, the color change (pink to yellow) only occurred in C. molybdites, indicating that the primer amplification was successful, whereas for the 43 negative controls and the blank control, no cross-reaction was detected and the color of the reaction tubes remained pink. Thus, we could prove that the LAMP primer set designed in this study is C. molybdites-specific.

Sensitivity refers to the minimum amount of DNA that can be detected. For this purpose, we prepared a series of 10fold dilutions of the genomic DNA of C. molybdites, ranging from $10 \mathrm{ng}$ to $0.1 \mathrm{fg}$. According to the color change of the reaction tube, the limit of detection (LOD) of the LAMP method was $1 \mathrm{pg}$ (Figure 4). It is much lower than many traditional detection methods (Epis et al., 2010; Praphruet and Charerntantanakul, 2014). Simultaneously, we compared the LAMP sensitivity of $C$. molybdites without a loop primer. In the absence of a loop primer, the LOD of the LAMP method was $10 \mathrm{pg}$, indicating that the loop primer could enhance sensitivity (Supplementary Figure 1).

\section{Method Applicability Analysis}

Consider that, in practical applications, mushrooms are usually processed before being consumed by people. Furthermore, in clinical practice, we usually test the vomit or feces of patients with mushroom poisoning. When mushrooms are accidentally ingested and entered the stomach, their DNA would be further degraded by the digestive juices. In order to evaluate the feasibility of our method, LAMP assays were carried out in boiled and digested samples. As shown in Figure 5, the LAMP primer set designed in this study detected the $C$. molybdites from both single and mixture samples that had been boiled and digested, as well as in the mixtures with a $C$. molybdites content as low as $1 \%$. The blank controls were not reacted, and the LAMP primers reacted negatively to human saliva. In conclusion, the LAMP method could be successfully applied for the detection of processed and digested C. molybdites in both single and mixtures.

\section{DISCUSSION}

Mushroom gatherers may mistakenly identify mushrooms from the species of $C$. molybdites as edible, thus causing food poisoning. In this study, we established a LAMP method that is relatively simple and field-adaptable.

One of the challenges in establishing an efficient LAMP detection assay is the selection of species-specific amplification targets (Tomita et al., 2008). Mitochondrial DNA has been widely studied in species for the selection of target genes based on its multiple copies, matrilineal inheritance, and rapid evolution rate (Dulay et al., 2020; Mesic et al., 2020). Among these, the ITS gene has been proven to have high intraspecific differences and low interspecies variation. Schoch et al. (2012) tested six target regions (SSU, LSU, ITS, RPB1, RPB2, and MCM7) commonly used in the molecular identification of fungi. They proposed the use of ITS genes as the official fungal amplification target. This proposal was supported by the scientific community and later used as the Barcode of Life ${ }^{4}$ by the Consortium. ITS gene fragments are commonly used in the detection and identification of mushroom species (Garnica et al., 2016; Badotti et al., 2017; Wang S. et al., 2019). A rich and complete database is the basis for the design of specific primers. Fungi are a group of organisms that show great diversity in morphology, life cycle, and ecology; the current research on fungi is very limited. In

${ }^{4}$ http://barcodinglife.org/ 
TABLE 2 | Species identification of 44 mushrooms based on Sanger sequencing.

\begin{tabular}{|c|c|c|}
\hline Sample ID & Results of ITS (sequence similarity) & $\begin{array}{c}\text { GenBank } \\
\text { accession no. }\end{array}$ \\
\hline M1 & Chlorophyllum molybdites (100\%) & MW192451 \\
\hline M2 & Suillus bovinus (99.4\%) & MW192452 \\
\hline M3 & Gymnopus subnudus (99.73\%) & MW192453 \\
\hline M4 & Panaeolus subbalteatus (99.36\%) & MW192454 \\
\hline M5 & Leucoagaricus rubrotinctus (99.70\%) & MW192455 \\
\hline M6 & Macrolepiota dolichaula (99.72\%) & MW192456 \\
\hline M7 & Lactarius subbrevipes (99.81\%) & MW192457 \\
\hline M8 & Laccaria aurantia (99.85\%) & MW192458 \\
\hline M9 & Amanita griseofolia (100\%) & MW192459 \\
\hline M10 & Rhizocybe alba (98.32\%) & MW192460 \\
\hline M11 & Russula variata (99.33\%) & MW192461 \\
\hline M12 & Russula senecis (98.57\%) & MW192462 \\
\hline M13 & Amanita hemibapha (100\%) & MW192463 \\
\hline M14 & Boletus kauffmanii (99.32\%) & MW192464 \\
\hline M15 & Tylopilus neofelleus (100\%) & MW192465 \\
\hline M16 & Russula rosacea (99.68\%) & MW192466 \\
\hline M17 & Butyriboletus yicibus (99.87) & MW192467 \\
\hline M18 & Russula velenovskyi (100\%) & MW192468 \\
\hline M19 & Hydnellum caeruleum (99.73\%) & MW192469 \\
\hline M20 & Tricholoma saponaceum (98.35\%) & MW192470 \\
\hline M21 & Inocybe mixtilis (100\%) & MW192471 \\
\hline M22 & Hydnellum concrescens (98.52\%) & MW192472 \\
\hline M23 & Amanita spissacea (99.48\%) & MW192473 \\
\hline M24 & Tricholoma albobrunneum (100\%) & MW192474 \\
\hline M25 & Pleurotus eryngii (100\%) & MW192475 \\
\hline M26 & Flammulina filiformis (99.83\%) & MW192476 \\
\hline M27 & Lentinula edodes (100\%) & MW192477 \\
\hline M28 & Hypsizygus marmoreus (100\%) & MW192478 \\
\hline M29 & Tricholoma olivaceoluteolum (100\%) & MW192479 \\
\hline M30 & Amanita citrinoannulata (100\%) & MW192480 \\
\hline M31 & Russula crustosa (99.70\%) & MW192481 \\
\hline M32 & Hebeloma crustuliniforme (99.71\%) & MW192482 \\
\hline Мз3 & Tricholoma imbricatum (99.86\%) & MW192483 \\
\hline M34 & Amanita parvipantherina (100\%) & MW192484 \\
\hline M35 & Amanita verrucosivolva (100\%) & MW192485 \\
\hline M36 & Tricholoma matsutake (100\%) & MW192486 \\
\hline M37 & Amanita concentrica (99.86\%) & MW192487 \\
\hline M38 & Amanita sepiacea (98.90\%) & MW192488 \\
\hline M39 & Pleurotus ostreatus (100\%) & MW192489 \\
\hline M40 & Tylopilus microsporus (100\%) & MW192490 \\
\hline M41 & Inocybe rimosa (98.91\%) & MW192491 \\
\hline M42 & Amanita pseudovaginata (100\%) & MW192492 \\
\hline M43 & Russula sanguinea (99.31\%) & MW192493 \\
\hline M44 & Gymnopus dryophilus (100\%) & MW537049 \\
\hline
\end{tabular}

molecular biology studies on fungal species, a large number of species are only represented by a single strain or specimen, and thus, nucleotide variation data for within-species and closesource species comparisons are lacking (Xu, 2016). Information on such variations is essential to distinguish closely related species (Xu, 2016). As one of the fungi with large species abundance, mushrooms are also faced with the problem of lack of genetic information. Therefore, we first examined the integrity and availability of nucleic acid sequences of Chlorophyllum before designing the specific C. molybdites LAMP primer. According to the literature, there are about 20 species of Chlorophyllum recorded in the world (Vellinga, 2002; Ge et al., 2018). We searched the ITS sequences of these species through NCBI, and all the species were successfully searched. Therefore, in the present study, we selected the ITS gene as the molecular marker to design species-specific LAMP primer sets.

In the selection of binding sites for LAMP primers, sufficient numbers of mismatches with non-target species should be confirmed. For example, rainbow trout (Oncorhynchus mykiss) LAMP primers designed by Xiong et al. (2020) had a total of 43 mismatch sites against their near-source species (Salmo salar, Oncorhynchus gorbuscha, Oncorhynchus tshawytscha, Oncorhynchus keta, Oncorhynchus masou, and Oncorhynchus nerka), resulting in high specificity for the rainbow trout. The primer set designed in this study had several mismatches against other species of Chlorophyllum at the $3^{\prime}$ end of C. mol-F3/B3 and the $5^{\prime}$ end of C. mol-F1c/B1c regions (Figure 1), demonstrating the high specificity of the $C$. molybdites primer set.

The sensitivity of LAMP detection is usually 10-100 times higher than that of conventional PCR assays (Wang et al., 2015). The method proposed in the present study proved to have high sensitivity and could detect $1 \mathrm{pg}$ of $C$. molybdites DNA. This conclusion was confirmed by multiple independent repeated experiments (Figure 4). In related studies, the LAMP detection method established by Chen Zuohong's research team from Hunan Normal University for lethal Amanita species had a detection limit of 10 pg (He et al., 2019). The sensitivity of the LAMP assay might be affected by many factors. It has been reported that the addition of loop primers could increase the sensitivity and specificity of the LAMP reaction (Nagamine et al., 2002). Nagamine et al. (2002) found that significant amplification of $10^{3}$ lambda DNA could be observed with loop primers. While removing loop primers, the signal was dispersed among $10^{3}$ copies within 52-92 min, which proved that significant DNA amplification required at least $10^{4}$ copies, indicating a higher sensitivity of the LAMP reaction with loop primers. The detection limit of C. molybdites in our study also confirmed this. In addition, some researchers found that the amount of DNA templates larger than $200 \mathrm{ng}$ had an inhibitory effect on the LAMP reaction (Njiru et al., 2008). In the current study, when the content of $C$. molybdites DNA added to the reaction system was $100 \mathrm{ng}$, the LAMP reaction was inhibited, and the color of the final product was an intermediate color of pink and yellow instead of yellow, which was consistent with the findings of Niiru et al. (2008) (results were not shown in Figure 4). This is presumably due to the complexity of the mushroom matrix, which contains many polysaccharides and polyphenols (Abubakar et al., 2018). Polyphenols can be oxidized after a long time, with irreversibly bound proteins and nucleic acids forming high molecular weight complexes (Newbury and Possingham, 1977). Polysaccharides can hinder the activity of polymerase and inhibit the amplification reaction of LAMP (Fang et al., 1992). Therefore, during detection, attention should be 


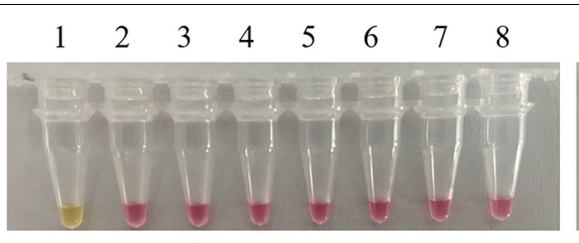

$\begin{array}{llllllll}17 & 18 & 19 & 20 & 21 & 22 & 23 & 24\end{array}$

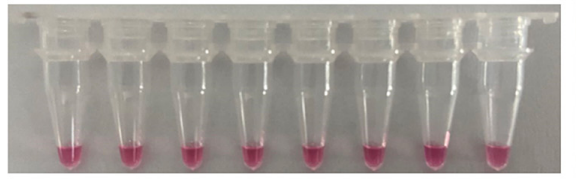

$\begin{array}{llllllll}33 & 34 & 35 & 36 & 37 & 38 & 39 & 40\end{array}$

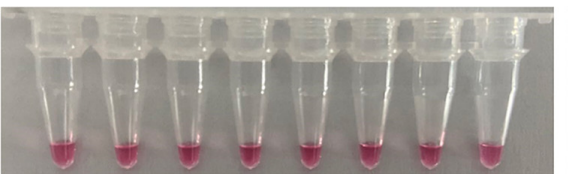

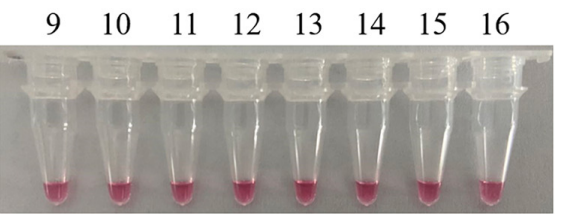

$\begin{array}{llllllll}25 & 26 & 27 & 28 & 29 & 30 & 31 & 32\end{array}$

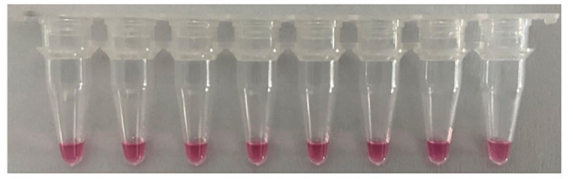

$\begin{array}{lllll}41 & 42 & 43 & 44 & 0\end{array}$

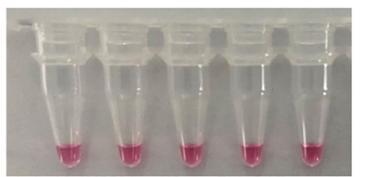

FIGURE 3 | Specificity test of the LAMP primer set for Chlorophyllum molybdites designed in this study. The set of primer was amplified for the detection of C. molybdites and 43 other non-target species. 1: C. molybdites; 2: Suillus bovinus; 3: Gymnopus subnudus; 4: Panaeolus subbalteatus; 5: Leucoagaricus rubrotinctus; 6: Macrolepiota dolichaula; 7: Lactarius subbrevipes; 8: Laccaria aurantia; 9: Amanita griseofolia; 10: Rhizocybe alba; 11: Russula variata; 12: Russula senecis; 13: Amanita hemibapha; 14: Boletus kauffmanii; 15: Tylopilus neofelleus; 16: Russula rosacea; 17: Butyriboletus yicibus; 18: Russula velenovskyi; 19: Hydnellum caeruleum; 20: Tricholoma saponaceum; 21: Inocybe mixtilis; 22: Hydnellum concrescens; 23: Amanita spissacea; 24: Tricholoma albobrunneum; 25: Pleurotus eryngii; 26: Flammulina filiformis; 27: Lentinula edodes; 28: Hypsizygus marmoreus; 29: Tricholoma olivaceoluteolum; 30: Amanita citrinoannulata; 31: Russula crustosa; 32: Hebeloma crustuliniforme; 33: Tricholoma imbricatum; 34: Amanita parvipantherina; 35: Amanita verrucosivolva; 36: A. verrucosivolva; 37: Amanita concentrica; 38: Amanita sepiacea; 39: Pleurotus ostreatus; 40: Tylopilus microsporus; 41: Inocybe rimosa; 42: Amanita pseudovaginata; 43: Russula sanguinea; 44: Gymnopus dryophilus; 0: $\mathrm{ddH}_{2} \mathrm{O}$.

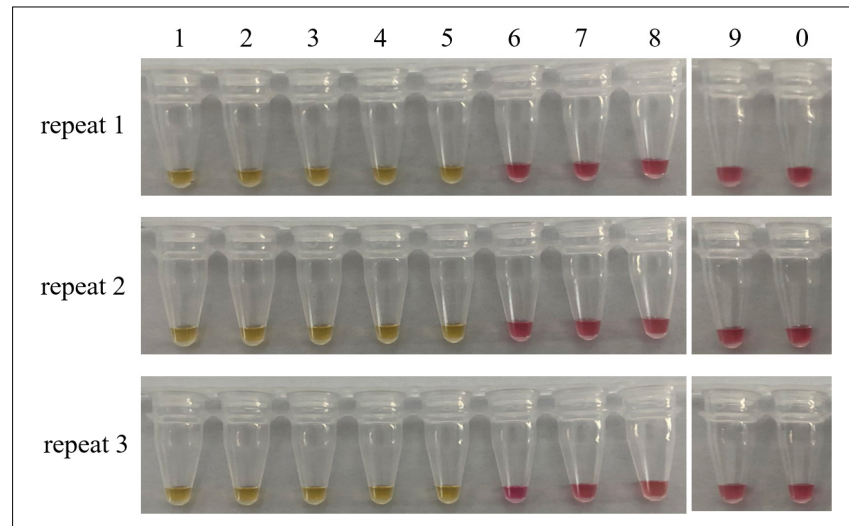

FIGURE 4 | Sensitivity test of the LAMP assay. Minimum amount of detectable DNA of the LAMP method established in this study was evaluated by using a series of $C$. molybdites DNA dilutions. For each reaction tube, we carried out three replicates. The color changed to yellow indicated positive amplification. 1: $10 \mathrm{ng} ; 2$ : 1 ng; 3: 0.1 ng; 4: $0.01 \mathrm{ng} ; 5: 1$ pg; 6: 0.1 pg; 7: $0.01 \mathrm{pg} ;$ 8: $1 \mathrm{fg}$; 9: $0.1 \mathrm{fg}$; 0: $\mathrm{ddH}_{2} \mathrm{O}$.

paid to controlling the content of the DNA template in the reaction system.

Visualization of LAMP amplification results is more conducive to rapid field detection. A variety of LAMP rapid visual inspection methods have been developed in recent years. Among them, the most commonly used methods are turbidity

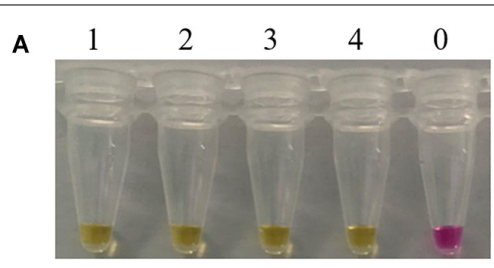

B

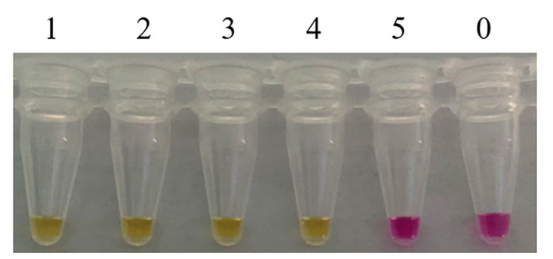

FIGURE 5 | Suitability assessment of the LAMP assay. Feasibility analysis of the LAMP method in on-site detection was evaluated by simulating the course of processing and digestion of mushrooms. (A) LAMP assay used for the detection of boiled Chlorophyllum molybdites. 1: C. molybdites; $2: 50 \%$ C. molybdites $+50 \%$ Pleurotus eryngii; 3 : $10 \%$ C. molybdites $+90 \%$ P. eryngii; 4: $1 \%$ C. molybdites $+99 \%$ P. eryngii; 0: dd $_{2} \mathrm{O}$. (B) LAMP assay used for the detection of digested C. molybdites after boiled. 1: C. molybdites; 2: $50 \%$ C. molybdites $+50 \%$ P. eryngii; 3: $10 \%$ C. molybdites $+90 \%$ P. eryngii; 4: $1 \%$ C. molybdites $+99 \%$ P. eryngii; 5: human saliva; 0: $\mathrm{ddH}_{2} \mathrm{O}$.

detection and color change (Tomita et al., 2008; Denschlag et al., 2013). The turbidity detection method is simple and cost-saving. 
However, this method has low detection sensitivity. When the concentration of the amplified product is low, the result is not always visible (Njiru et al., 2008). The detection method based on a color change has proven to be an effective alternative method. Many metal ion indicators, such as hydroxyl naphthol blue (HNB) and calcein, are used as indicative dyes for LAMP amplification (Pang et al., 2019; Chen et al., 2020; Wigmann et al., 2020). Whether the reaction has occurred can be determined according to the color change in the tubes. The reaction results are visible to the naked eye, and furthermore, there is no need to open the tubes during the reaction process, which can effectively avoid aerosol contamination and the probability of false positives (Goto et al., 2009). However, the LAMP amplification products are not obvious in terms of color changes with the HNB and calcein dyes. The WarmStart ${ }^{\circledR}$ Colorimetric LAMP 2X Master Mix (New England Biolabs, United States) used in this study contains a visible $\mathrm{pH}$ indicator. During the LAMP reaction, the $\mathrm{pH}$ of the reaction solution tends to decrease because of the production of protons and the subsequent enhancement of DNA polymerase activity, such that the color of the solution changes from pink to yellow. The color change is obvious, and the reaction result is visible to the naked eye under daylight conditions, which is consistent with the results of Daskou et al. (2019). In addition, considering that researchers are affected by subjective preference, we put the reaction tube on white paper after amplification and used the white color of the paper as a control to ensure that the difference in color between positive and negative tubes became more pronounced. Furthermore, double-blind experiments were carried out in the laboratory, and all observers had no disagreement with the test results. The LAMP visual detection method established in this study will provide ideas for the further development of microfluidic chips and integrated on-site rapid detection methods.

For mushroom poisoning incidents, in the examination of clinical patients and forensic investigation, the detection of vomit, feces, and stomach contents is required. In order to ensure the feasibility of this method, we simulated the process of mushroom processing and digestion in human gastric juice. In this study, we successfully detected C. molybdites in the mixed mushrooms digested with artificial gastric juice. High temperature, pressure, and the digestion of gastric juice may cause some degree of genomic DNA degradation and fragmentation. Previous studies have confirmed that DNA degradation would not affect the detection of fragments shorter than 300 bp (Goldstein and Desalle, 2003). Gausterer et al. (2012) used direct PCR to investigate the stomach contents in a forensic case and successfully detected the digested yew plant. Others had proved that DNA was still present in traditional food processing styles, and it could be amplified in dried mushrooms and even be incubated by gastric juices (Gausterer et al., 2014). The LAMP method for C. molybdites detection established in this study had the same conclusion. In conclusion, our study provided an evidence that the LAMP assay could be used for the rapid and sensitive detection of $C$. molybdites in various matrices, including raw, boiled, and digested mushrooms with single or mixed species.

\section{CONCLUSION}

According to the results of this present study, molecular biology methods focused on nucleic acid are of great necessity to detect mushrooms of C. molybdites. More specifically, the LAMP method targeting the mitochondrial ITS gene was established for the on-site rapid detection of C. molybdites. High specificity and sensitivity (the LOD of detectable DNA was $1 \mathrm{pg}$ ) for the LAMP method were confirmed in the present study. Finally, the established method was successfully applied to the boiled and digested mushroom mixtures. These characteristics indicated that the LAMP assay for species-specific detection was useful for identifying $C$. molybdites in clinical treatment and forensic analysis.

\section{DATA AVAILABILITY STATEMENT}

The original contributions presented in the study are included in the article/Supplementary Material, further inquiries can be directed to the corresponding author/s.

\section{AUTHOR CONTRIBUTIONS}

AC conceived of and designed the experiments. $\mathrm{ZZ}$ and XZ provided some Chlorophyllum molybdites materials and identified the species. ET completed the morphological identification of the mushroom samples. HL and JZ completed the analysis of ITS DNA sequences of all mushrooms. NW, JG, WY, and RX carried out the LAMP assay. NW and AC wrote the manuscript. All authors contributed to the article and approved the submitted version.

\section{FUNDING}

This study was funded by the National Key R\&D Program of China (No. 2019YFC1604700).

\section{SUPPLEMENTARY MATERIAL}

The Supplementary Material for this article can be found online at: https://www.frontiersin.org/articles/10.3389/fmicb. 2021.638315/full\#supplementary-material

Supplementary Figure 1 | Sensitivity test of the Chlorophyllum molybdites LAMP assay without a loop primer. Minimum amount of detectable DNA of the LAMP method established in this study was evaluated by using a series of $C$. molybdites DNA dilutions. The color changing to yellow indicated positive amplification. 1: 10 ng; 2: 1 ng; 3: 0.1 ng; 4: 0.01 ng; 5: 1 pg; 6: 0.1 pg; 7: 0.01 pg; 8: 1 fg; 9: 0.1 fg; $0: \mathrm{ddH}_{2} \mathrm{O}$. 


\section{REFERENCES}

Abubakar, B. M., Izham, N. H. M., Salleh, F. M., Shamsir, S., and Wagiran, A. (2018). Comparison of different DNA extraction methods from leaves and roots of Eurycoma longifolia Plant. Adv. Sci. Lett. 24, 3641-3645. doi: 10.1166/asl. 2018.11456

Ahmed, W. H. A., Gonmori, K., Suzuki, M., Watanabe, K., and Suzuki, O. (2010). Simultaneous analysis of $\alpha$-amanitin, $\beta$-amanitin, and phalloidin in toxic mushrooms by liquid chromatography coupled to time-of-flight mass spectrometry. Forensic. Toxicol. 28, 69-76. doi: 10.1007/s11419-0090089-7

Badotti, F., de Oliveira, F. S., Garcia, C. F., Martins Vaz, A. B., Camargos Fonseca, P. L., Nahum, L. A., et al. (2017). Effectiveness of ITS and sub-regions as DNA barcode markers for the identification of Basidiomycota (Fungi). BMC Microbiol. 17:42. doi: 10.1186/s12866-017-0958-x

Becherer, L., Borst, N., Bakheit, M., Frischmann, S., and Stetten, F. V. (2020). Loop-mediated isothermal amplification (LAMP) - review and classification of methods for sequence-specific detection. Anal. Methods 12, 717-746. doi: 10.1039/C9AY02246E

Bodulev, O. L., and Sakharov, I. Y. (2020). Isothermal nucleic acid amplification techniques and their use in bioanalysis. Biochemistry 85, 147-166. doi: 10.1134/ S0006297920020030

Chen, Y., Zhang, L., Miao, J., and Li, J. (2020). Dye-sensitized halide perovskite: a case study on calcein dye. Dyes. Pigm. 181:108608. doi: 10.1016/j.dyepig.2020. 108608

Chen, Z., Zhang, P., and Zhang, Z. (2014). Investigation and analysis of 102 mushroom poisoning cases in Southern China from 1994 to 2012. Fungal Divers. 64, 123-131. doi: 10.1007/s13225-013-0260-7

Daskou, M., Tsakogiannis, D., Dimitriou, T. G., Amoutzias, G. D., Mossialos, D., Kottaridi, C., et al. (2019). WarmStart colorimetric LAMP for the specific and rapid detection of HPV16 and HPV18 DNA. J. Virol. Methods 270, 87-94. doi: 10.1016/j.jviromet.2019.04.023

Denschlag, C., Vogel, R. F., and Niessen, L. (2013). Hyd5 gene based analysis of cereals and malt for gushing-inducing Fusarium spp. by real-time LAMP using fluorescence and turbidity measurements. Int. J. Food Microbiol. 162, 245-251. doi: 10.1016/j.ijfoodmicro.2013.01.019

Dulay, R. M. R., Cabrera, E. C., Kalaw, S. P., and Reyes, R. G. (2020). Nucleotide sequencing and identification of wild mushrooms from the Philippines. Biocatal. Agric. Biotechnol. 27:101666. doi: 10.1016/j.bcab.2020.10 1666

Epis, S., Matinato, C., Gentili, G., Varotto, F., Bandi, C., and Sassera, D. (2010). Molecular detection of poisonous mushrooms in different matrices. Mycologia 102, 747-754. doi: 10.3852/09-124

Fang, G., Hammar, S., and Grumet, R. (1992). A quick and inexpensive method for removing polysaccharides from plant genomic DNA. BioTechniques 13, 52-54, 56. doi: 10.1002/bmc.1130060412

Garnica, S., Schoen, M. E., Abarenkov, K., Riess, K., Liimatainen, K., Niskanen, T., et al. (2016). Determining threshold values for barcoding fungi: lessons from Cortinarius (Basidiomycota), a highly diverse and widespread ectomycorrhizal genus. FEMS Microbiol. Ecol. 92:fiw045. doi: 10.1093/femsec/fiw045

Gausterer, C., Penker, M., Krisai-Greilhuber, I., Stein, C., and Stimpfl, T. (2014). Rapid genetic detection of ingested Amanita phalloides. Forensic. Sci. Int. Genet. 9, 66-71. doi: 10.1016/j.fsigen.2013.11.002

Gausterer, C., Stein, C., and Stimpfl, T. (2012). Application of direct PCR in a forensic case of yew poisoning. Int. J. Legal. Med. 126, 315-319. doi: 10.1007/ s00414-011-0607-0

Ge, Z. W., Jacobs, A., Vellinga, E. C., Sysouphanthong, P., van der Walt, R., Lavorato, C., et al. (2018). A multi-gene phylogeny of Chlorophyllum (Agaricaceae, Basidiomycota): new species, new combination and infrageneric classification. MycoKeys 32, 65-90. doi: 10.3897/mycokeys.32.23831

Goldstein, P. Z., and Desalle, R. (2003). Calibrating phylogenetic species formation in a threatened insect using DNA from historical specimens. Mol. Ecol. 12, 1993-1998. doi: 10.1046/j.1365-294x.2003.01860.x

Goto, M., Honda, E., Ogura, A., Nomoto, A., and Hanaki, K. (2009). Colorimetric detection of loop-mediated isothermal amplification reaction by using hydroxy naphthol blue. Biotechniques 46, 167-172. doi: 10.2144/000113072

Haijiao, L., Hongshun, Z., Yizhe, Z., Kaiping, Z., Jing, Z., Yu, Y., et al. (2020). Mushroom Poisoning Outbreaks - China, 2019. China CDC Weekly 2, 19-24.
He, Z., Su, Y., Li, S., Long, P., Zhang, P., and Chen, Z. (2019). Development and evaluation of isothermal amplification methods for rapid detection of lethal Amanita Species. Front. Microbiol. 10:1523. doi: 10.3389/fmicb.2019.01523

Hebert, P., Cywinska, A., Ball, S. L., and Dewaard, J. (2003). Biological identification through DNA barcodes. Proc. R. Soc. Lond. B 270, 313-321.

Jia, B., Li, X., Liu, W., Lu, C., Lu, X., Ma, L., et al. (2019). GLAPD: whole genome based LAMP primer design for a set of target genomes. Front. Microbiol. 10:2860. doi: 10.3389/fmicb.2019.02860

Kondo, K., Sakata, K., Kato, R., Sugano, Y., Takeuchi, S., and Sato, M. (2019). Qualitative real-time PCR method for poisonous Entoloma rhodopoliumrelated species in Japan: real-time PCR method for entoloma mushrooms. Food Hygiene Saf. Sci. 60, 144-150. doi: 10.3358/shokueishi.60.144

Lee, P. L. M. (2017). DNA amplification in the field: move over PCR, here comes LAMP. Mol. Ecol. Resour. 17, 138-141. doi: 10.1111/1755-0998.12548

Lei, S., Shi, P., Wu, W., Xia, B., Fu, X., Wan, Y., et al. (2020). Extensive screening of cyclopeptide toxins in mushrooms by ultra-high-performance liquid chromatography coupled with quadrupole-Orbitrap mass spectrometry. Food Chem. 329:127146. doi: 10.1016/j.foodchem.2020.127146

Li, H., Sun, C., Qiao, L., Zhang, Y., Sun, J., Zhang, H., et al. (2016). Species identification, poisoning symptoms and medical treatment of Chlorophyllum molybdites. Chin. J. Emerg. Med. 25, 739-742.

Lv, X., Wang, L., Zhang, J., Zeng, H., Chen, X., Shi, L., et al. (2020). Rapid and sensitive detection of VBNC Escherichia coli O157: H7 in beef by PMAxx and real-time LAMP. Food Control 115:107292. doi: 10.1016/j.foodcont.2020. 107292

Mesic, A., Samec, D., Jadan, M., Bahun, V., and Tkalcec, Z. (2020). Integrated morphological with molecular identification and bioactive compounds of 23 Croatian wild mushrooms samples. Food Biosci. 37:100720. doi: 10.1016/j.fbio. 2020.100720

Minekus, M., Alminger, M., Alvito, P., Ballance, S., Bohn, T., Bourlieu, C., et al. (2014). A standardised static in vitro digestion method suitable for food - an international consensus. Food Funct. 5, 1113-1124. doi: 10.1039/c3fo60702j

Nagamine, K., Hase, T., and Notomi, T. (2002). Accelerated reaction by loopmediated isothermal amplification using loop primers. Mol. Cell. Probes 16, 223-229. doi: 10.1006/mcpr.2002.0415

Newbury, H. J., and Possingham, J. V. (1977). Factors affecting the extraction of intact ribonucleic acid from plant tissues containing interfering phenolic compounds. Plant Physiol. 60, 543-547. doi: 10.1104/pp.60.4.543

Njiru, Z. K., Mikosza, A. S. J., Armstrong, T., Enyaru, J. C., Ndung'u, J. M., and Thompson, A. R. C. (2008). Loop-mediated isothermal amplification (LAMP) method for rapid detection of Trypanosoma brucei rhodesiense. PLoS Negl. Trop. Dis. 2:e147. doi: 10.1371/journal.pntd.0000147

Notomi, T., Okayama, H., Masubuchi, H., Yonekawa, T., Watanabe, K., Amino, N., et al. (2000). Loop-mediated isothermal amplification of DNA. Nucleic Acids Res. 28:e63. doi: 10.1093/nar/28.12.e63

Numanoglu Cevik, Y. (2020). The effect of a high-resolution accurate mass spectrometer on simultaneous multiple mushroom toxin detection. Gümüşhane Üniversitesi Fen Bilimleri Enstitüsü Dergisi 10, 878-886. doi: 10.17714/ gumusfenbil.680816

Pang, B., Yao, S., Xu, K., Wang, J., Song, X., Mu, Y., et al. (2019). A novel visual-mixed-dye for LAMP and its application in the detection of foodborne pathogens. Anal. Biochem. 574, 1-6. doi: 10.1016/j.ab.2019.03.002

Praphruet, R., and Charerntantanakul, W. (2014). Rapid identification of poisonous mushroom species by Real-time PCR-based technique. J. Agric. Res. Extens. 31, 45-53.

Qiu, X., Chen, D., Wang, X., Zhou, H., Hou, X., Zhang, J., et al. (2019). A novel isothermal amplification-based method for detection of Corynebacterium striatum. J. Microbiol. Methods 164:105675.

Schoch, C. L., Seifert, K. A., Huhndorf, S., Robert, V., Spouge, J. L., Levesque, C. A., et al. (2012). Nuclear ribosomal internal transcribed spacer (ITS) region as a universal DNA barcode marker for Fungi. Proc. Natl. Acad. Sci. U.S.A. 109, 6241-6246. doi: 10.1073/pnas.1117018109

Soltaninejad, K. (2018). Outbreak of mushroom poisoning in iran: april-may, 2018. Int. J. Occup. Environ. Med. 9, 152-156. doi: 10.15171/ijoem.2018.1380

Sugano, Y., Sakata, K., Nakamura, K., Noguchi, A., Fukuda, N., Suzuki, T., et al. (2017). Rapid identification method of Omphalotus japonicus by polymerase chain reaction-restriction fragment length polymorphism (PCR-RFLP). J. Food Hygienic Soc. Jan. 58, 113-123. doi: 10.3358/shokueishi.58.113 
Sun, J., Li, H. J., Zhang, H. S., Zhang, Y. Z., Xie, J. W., Ma, P. B., et al. (2018). Investigating and analyzing three cohorts of mushroom poisoning caused by Amanita exitialis in Yunnan. China. Hum. Exp. Toxicol. 37, 665-678. doi: 10.1177/0960327117721960

Tomita, N., Mori, Y., Kanda, H., and Notomi, T. (2008). Loop-mediated isothermal amplification (LAMP) of gene sequences and simple visual detection of products. Nat Protoc. 3, 877-882. doi: 10.1038/nprot. 2008.57

Tripathi, P., Upadhyay, N., and Nara, S. (2018). Recent advancements in lateral flow immunoassays: a journey for toxin detection in food. Crit. Rev. Food Sci. Nutr. 58, 1715-1734. doi: 10.1080/10408398.2016.12 76048

Vaagt, F., Haase, I., and Fischer, M. (2013). Loop-mediated isothermal amplification (LAMP)-based method for rapid mushroom species identification. J. Agric. Food Chem. 61, 1833-1840. doi: 10.1021/jf304824b

Vellinga, E. C. (2002). New combinations in Chlorophyllum. Mycotaxon 83, 415417. doi: 10.1109/TAES.2007.4441751

Wang, S., Guo, H., Li, J., Li, W., Wang, Q., and Yu, X. (2019). Evaluation of five regions as DNA barcodes for identification of Lepista species (Tricholomataceae, Basidiomycota) from China. Peerj 7:e7307. doi: 10.7717/peerj.7307

Wang, W.-T., Han, C., Sun, Y.-M., Chen, Z.-H., Fang, K., Huang, W., et al. (2019). Activation of the lysosome-associated membrane protein LAMP5 by DOT1L serves as a bodyguard for MLL fusion oncoproteins to evade degradation in leukemia. Clin. Cancer. Res. 25, 2795-2808. doi: 10.1158/1078-0432.Ccr-181474

Wang, X., Seo, D. J., Lee, M. H., and Choi, C. (2014). Comparison of conventional PCR, Multiplex PCR, and loop-mediated isothermal amplification assays for rapid detection of Arcobacter species. J. Clin. Microbiol. 52, 557-563. doi: 10. 1128/jcm.02883-13

Wang, X.-R., Wu, L.-F., Wang, Y., Ma, Y.-Y., Chen, F.-H., and Ou, H.-L. (2015). Rapid detection of Staphylococcus aureus by loop-mediated isothermal amplification. Appl. Biochem. Biotechnol. 175, 882-891. doi: 10.1007/s12010014-1328-x

White, T. J., Bruns, T., Lee, S., and Taylor, J. (1989). “Amplification and direct sequencing of fungal ribosomal RNA genes for phylogenetics," in PCR Protocols: A Guide to Methods and Applications, eds M. Innis, D. Gelfand, J. Sninsky, T. White, (New York: Academic Press, Inc.), 315-322. doi: 10.1016/B978-0-12372180-8.50042-1

Wigmann, E. F., Meyer, K., Cendoya, E., Maul, R., Vogel, R. F., and Niessen, L. (2020). A loop-mediated isothermal amplification (LAMP) based assay for the rapid and sensitive group-specific detection of fumonisin producing Fusarium spp. Int. J. Food Microbiol. 325:108627. doi: 10.1016/j.ijfoodmicro.2020.108627

Wu, F., Zhou, L.-W., Yang, Z.-L., Bau, T., Li, T.-H., and Dai, Y.-C. (2019). Resource diversity of Chinese macrofungi: edible, medicinal and poisonous species. Fungal Divers. 98, 1-76. doi: 10.1007/s13225-019-00432-7

Wurita, A., Hasegawa, K., Konno, K., Hashimoto, K., Gonmori, K., Minakata, K., et al. (2019). Quantification of clitidine in caps and stems of poisonous mushroom Paralepistopsis acromelalga by hydrophilic interaction liquid chromatography-tandem mass spectrometry. Forensic. Toxicol. 37, 378-386. doi: 10.1007/s11419-019-00470-5

Xiong, X., Huang, M., Xu, W., Li, Y., Cao, M., and Xiong, X. (2020). Rainbow trout (Oncorhynchus mykiss) identification in processed fish products using loopmediated isothermal amplification and polymerase chain reaction assays. J. Sci. Food Agric. 100, 4696-4704. doi: 10.1002/jsfa.10526

$\mathrm{Xu}$, J. (2016). Fungal DNA barcoding. Genome 59, 913-932. doi: 10.1139/gen2016-0046

Yamada, M., Tokumitsu, N., Saikawa, Y., Nakata, M., Asano, J., Miyairi, K., et al. (2012). Molybdophyllysin, a toxic metalloendopeptidase from the tropical toadstool, Chlorophyllum molybdites.. Biorg. Med. Chem. 20, 6583-6588. doi: 10.1016/j.bmc.2012.09.036

Yang, Z. (2013). Fungal taxonomy in the genomics era: opportunities and challenges. Mygosystema 32, 931-946.

Yao, S., Li, J. Q., Li, T., Liu, H. G., and Wang, Y. Z. (2018). Discrimination of Boletaceae mushrooms based on data fusion of FT-IR and ICP-AES combined with SVM. Int. J. Food Prop. 21, 270-281. doi: 10.1080/10942912.2018.1453838

Zhu, K., and Wang, D. (2018). Application of loop-mediated isothermal amplification technology in food safety inspection. J. Food Saf. Qual. 9, 47694774 .

Conflict of Interest: The authors declare that the research was conducted in the absence of any commercial or financial relationships that could be construed as a potential conflict of interest.

Copyright (c) 2021 Wang, Zhao, Gao, Tian, Yu, Li, Zhang, Xie, Zhao and Chen. This is an open-access article distributed under the terms of the Creative Commons Attribution License (CC BY). The use, distribution or reproduction in other forums is permitted, provided the original author(s) and the copyright owner(s) are credited and that the original publication in this journal is cited, in accordance with accepted academic practice. No use, distribution or reproduction is permitted which does not comply with these terms. 